\title{
Mariage et cadre de vie familiale dans une paroisse ouvrière montréalaise : Sainte-Brigide, 1900-1914
}

\section{Lucia Ferretti}

Volume 39, numéro 2, automne 1985

Histoire de la famille

URI : https://id.erudit.org/iderudit/304351ar

DOI : https://doi.org/10.7202/304351ar

Aller au sommaire du numéro

Éditeur(s)

Institut d'histoire de l'Amérique française

ISSN

0035-2357 (imprimé)

1492-1383 (numérique)

Découvrir la revue

Citer cet article

Ferretti, L. (1985). Mariage et cadre de vie familiale dans une paroisse ouvrière montréalaise : Sainte-Brigide, 1900-1914. Revue d'histoire de l'Amérique française, 39(2), 233-251. https://doi.org/10.7202/304351ar d'utilisation que vous pouvez consulter en ligne. 


\title{
MARIAGE ET CADRE DE VIE FAMILIALE DANS UNE PAROISSE OUVRIERE MONTRÉALAISE: SAINTE-BRIGIDE, 1900-1914 ${ }^{1}$
}

\author{
LUCIA FERRETTI \\ Département d'histoire \\ Université du Québec à Montréal
}

\section{Introduction}

La paroisse Sainte-Brigide peut être considérée comme un microcosme des transformations économiques, sociales et culturelles qui bouleversent les quartiers populaires de Montréal durant la seconde moitié du 19e siècle et jusqu'à la première guerre mondiale.

Fondée en 1867 comme celle de Saint-Vincent-de-Paul, SainteBrigide est avec elle la plus ancienne paroisse du quartier Sainte-Marie, dont elle occupe par ailleurs le coeur industriel. Elle couvre à l'origine un vaste territoire d'environ deux milles carrés. Mais l'augmentation considérable et continuelle de la population du secteur a entraîné la création de nouvelles paroisses et, en conséquence, le démembrement de Sainte-Brigide. En 1900, elle ne dessert plus qu'un seul mille carré, l'envers du mille carré doré si l'on peut dire, circonscrit d'une part par le fleuve et la rue Logan et d'autre part par les rues Panet et Colborne (aujourd'hui De Lorimier). En outre, en 1912, une toute petite partie du territoire, à la pointe nord-ouest, passe sous la juridiction de la nouvelle paroisse de Sainte-Catherine-d'Alexandrie.

Depuis sa fondation, la paroisse s'est urbanisée au même rythme que Montréal, sinon à un rythme encore plus rapide, ainsi que l'ont montré les travaux de Carmen Soucy-Roy pour l'ensemble du quartier Sainte-Marie ${ }^{2}$. Sa physionomie se modifie considérablement à cette époque. De nouveaux établissements manufacturiers ne cessent de s'y implanter, faisant de Sainte-Brigide une paroisse industrielle typique. L'essor démographique est tel que ce n'est qu'au prix de démembrements successifs que la paroisse peut se maintenir autour de 12000 habitants entre 1867 et $1914^{3}$. La construction domiciliaire suit ce rythme

\footnotetext{
1 Je tiens à remercier Messieurs Jean-Paul Bernard et Stanley Bréhaut Ryerson, directeurs du mémoire de maîtrise dont cet article est tiré, et Madame Micheline Dumont, qui a lu la première version de ce texte, pour les conseils et le soutien qu'ils m'ont largement prodigués.

2 Carmen Soucy-Roy, Le quartier Sainte-Marie (1850-1900). Mémoire de maîtrise (histoire), Université du Québec à Montréal, 1977, $179 \mathrm{p}$.

3 L'évaluation de la population brigidaine s'est faite grâce à plusieurs sources. Pour 1880 et 1890, par les rôles d'évaluation des quartiers Sainte-Marie et Saint-Jacques; pour 1883, par les Archives de la Chancellerie de l'Archevêché de Montréal, 335-129, 884-2; pour les années 19001914, par les Prônes de Sainte-Brigide, cahier commençant le 11 octobre 1914, 1.
} 


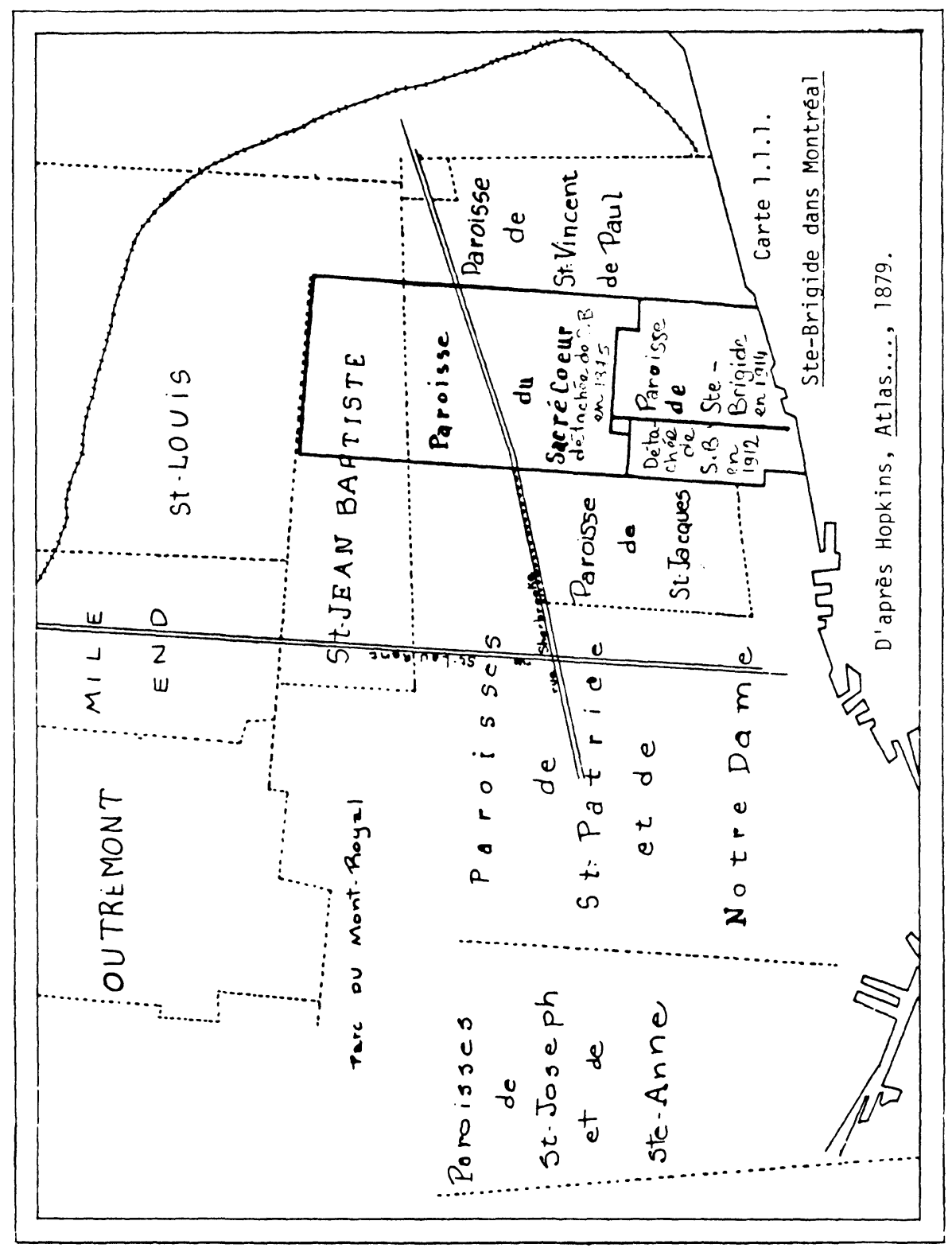


d'expansion. Le caractère ouvrier de la paroisse s'accentue de plus en plus, de même que son caractère francophone, malgré la présence, à ses confins oriental et méridional, d'une forte minorité irlandaise ${ }^{4}$. Artisanat, boutique et fabrique moderne coexistent sur une superficie aussi restreinte, de même que se côtoient urbains de plus vieille souche et nouveaux arrivés en ville. C'est ce qui rend la paroisse si intéressante pour une étude de certains aspects de l'univers culturel des travailleurs à l'époque de l'industrialisation accélérée de Montréal. C'est ce qui en fait aussi le lieu tout désigné pour une analyse du mariage et du cadre de vie familiale en milieu ouvrier.

Après avoir établi le caractère ouvrier de la population brigidaine, à l'aide d'indicateurs reliés au statut professionnel et à la provenance géographique des conjoints et à la cohésion sociale de leur milieu, on analysera les différentes composantes du cadre de vie familiale, puis on décrira la cérémonie nuptiale. Pour procéder, une source principale a été utilisée, puis confrontée aux informations fournies par les recensements et les rôles d'évaluation. Il s'agit des registres de publications de bans, conservés exceptionnellement à Sainte-Brigide, pour les années 1905-1914. Ces registres fournissent la liste des promesses de mariage annoncées officiellement par le curé au prône dominical. La promesse de mariage est beaucoup plus détaillée que l'acte de mariage. Elle dévoile deux grandes catégories de renseignements: d'abord des informations de type sociologique, telles que le lieu de résidence et le lieu de naissance des conjoints, l'âge au mariage, la profession de l'époux dans presque tous les cas mais jamais celle de l'épouse, la profession des pères des conjoints, le lieu de résidence des parents, la mention de leur décès le cas échéant; puis des informations de type plus culturel, avec des détails sur la vie sociale des conjoints et la cérémonie du mariage et des indications sur le cadre de la vie familiale (présence ou non de pensionnaires, formes de la solidarité familiale).

Ces registres de publications de bans nous renvoient une image légèrement déformée de la réalité paroissiale. Ils concentrent en effet notre attention essentiellement sur les jeunes, au détriment de leurs parents et tout particulièrement au détriment de leur mère. Parmi ces jeunes, ils privilégient par ailleurs ceux qui se marient contre ceux qui demeurent célibataires. En outre, ils ne communiquent pas la liste complète des mariages impliquant au moins un travailleur ou une travailleuse résidant à Sainte-Brigide pour 1905-1914. En effet, étant donnée la coutume voulant que la cérémonie soit célébrée dans la paroisse de l'épouse, nous perdons, sauf rares exceptions, toutes les unions bénies entre un Brigidain et une résidente d'une autre paroisse. Nous disposons

$4 \quad$ Rôles d'évaluation, quartiers Sainte-Marie et Saint-Jacques, 1880 et 1890. 
de 1105 dossiers de mariages ouvriers ${ }^{5}$ ce qui représente $72 \%$ de tous les mariages qui ont effectivement impliqué un Brigidain ou une Brigidaine entre 1905 et 1914 . Les registres ont en effet notifié tous les mariages de Brigidaines et $44,1 \%$ de ceux qui concernent un Brigidain ${ }^{6}$.

Malgré ces réserves, on peut cependant estimer que les individus recensés sont assez représentatifs de l'ensemble de la population, tant pour l'origine géographique et la profession que pour l'ascendance sociale et professionnelle. En fournissant de nombreuses informations sociologiques et culturelles, ces registres contribuent de manière importante à l'amélioration de notre connaissance des travailleurs brigidains et montréalais du début du $20 \mathrm{e}$ siècle.

\section{1 - Une population ouvrière}

Entre 1905 et 1914 tout au moins, Sainte-Brigide est bien une paroisse ouvrière. Le relevé des professions des conjoints, de leurs origines géographiques, de même que l'analyse de l'hérédité du statut social $^{7}$ et de l'endogamie sociale le confirment à l'envi.

Un jeune Brigidain sur cinq $(20,7 \%)$ est journalier, six sur dix sont ouvriers proprement dits, surtout menuisiers, peintres, plombiers, charretiers, tailleurs et cordonniers, un sur huit est employé: au total, tout près de $90 \%$ des jeunes hommes de Sainte-Brigide sont des salariés. A peine trois sur cent tiennent un commerce ou une petite fabrique tandis que six sur cent pratiquent la médecine, le droit, le génie ou la comptabilité. L'habitude du curé de noter quasi-systématiquement la profession du futur dans la promesse de mariage ne laisse ainsi aucun doute sur le caractère ouvrier de la paroisse. D'ailleurs l'origine géographique des conjoints confirme cette première constatation.

Sur les 1358 personnes des classes laborieuses unies à SainteBrigide et dont on connaît la paroisse de baptême, 826 (soit 60,8\%) sont nées à Montréal ou dans une autre ville, contre $532(39,2 \%)$ à la campagne. La ville assure de plus en plus la reproduction sociale des travailleurs dès la fin du 19e siècle. Parmi les conjoints qui résident déjà en ville au moment du mariage, cette proportion monte d'ailleurs à tout près de $63 \%$, et lorsqu'on ventile les chiffres selon le sexe, on remarque qu'exactement les deux-tiers des épousées sont urbaines de

\footnotetext{
5 On entend par mariage ouvrier, un mariage impliquant au moins un travailleur ou une travailleuse.

61160 mariages ont été célébrés à Sainte-Brigide entre septembre 1905 et décembre 1914. Pour la même période, on possède 1192 publications de bans. Cela signifie qu'on a non seulement les publications de bans de toutes les unions bénies dans la paroisse, mais encore celles de trentedeux mariages célébrés à l'extérieur. Cf. Registres de publications de bans, paroisse SainteBrigide, 1905-1914, et Registres de mariages, paroisse Sainte-Brigide, 1905-1914.

C'est Yves Lequin qui le premier à utilisé ce concept dans le chapitre 5, «La naissance et le métier», de son ouvrage sur Les ouvriers de la région lyonnaise (1848-1914), tome I : La formation de la classe ouvrière régionale (Lyon, Presses universitaires de Lyon, 1977), 573 p.
} 
naissance contre $58,1 \%$ de leurs compagnons. Cet écart de plus de huit points est très significatif si l'on considère que c'est surtout par les femmes que se transmettent les valeurs culturelles enseignées les premières et assimilées durant la petite enfance. Peut-on penser qu'au début du $20 \mathrm{e}$ siècle, les conditions de base sont déjà réunies pour que se constitue un univers culturel propre aux travailleurs, adapté à la vie urbaine bien que toujours tributaire d'antécédents ruraux?

Mais il ne suffit pas de déterminer grossièrement le lieu d'origine. Les registres de publications de bans permettent de cerner de façon plus précise quelles régions, dans la province, fournissent le plus de travailleurs et de travailleuses à la paroisse Sainte-Brigide. L'île et la région de Montréal, particulièrement les Basses-Laurentides et l'immédiate rive-sud déversent de gros contingents dans la métropole et dans la paroisse. La ville et la région de Québec (comtés de Québec, Lévis, Portneuf, Montmorency, Montmagny) de même que la ville et la région de Trois-Rivières (comtés de Champlain, Saint-Maurice et Nicolet) les

TABLEAU 1

Professions des hommes mariés à

Ste-Brigide: 1905-1914

\begin{tabular}{|l|r|r|r|r|r|r|r|r|}
\hline & \multicolumn{2}{|c|}{ Résidant à S.B. } & \multicolumn{2}{c|}{$\begin{array}{c}\text { Rés. à Mtl sauf } \\
\text { S.B. }\end{array}$} & \multicolumn{2}{|c|}{$\begin{array}{c}\text { Résidant hors } \\
\text { de Mtl } \\
\text { (villes et } \\
\text { campagnes) }\end{array}$} & \multicolumn{2}{|c|}{ TOTAL } \\
Nbre & Nbre & $\%$ & Nbre & $\%$ & Nbre & $\%$ \\
\hline $\begin{array}{l}\text { 1- Commerçants et } \\
\text { Industriels }\end{array}$ & 16 & 3,6 & 40 & 7,3 & 12 & 11,2 & 68 & 6.2 \\
\hline $\begin{array}{l}\text { 2- Professions } \\
\text { libérales et } \\
\text { assimilées }\end{array}$ & 26 & 5,9 & 31 & 5,7 & 6 & 5,6 & 63 & 5,7 \\
\hline $\begin{array}{l}\text { 3- Employés du } \\
\text { commerce et des } \\
\text { services }\end{array}$ & 53 & 12,0 & 86 & 15,7 & 15 & 14,0 & 154 & 14,1 \\
\hline 4- Artisans et Artistes & 4 & 0,9 & 19 & 3,5 & 2 & 1,9 & 25 & 2,3 \\
\hline 5- Cultivateurs & 0 & - & 0 & - & 19 & 17,7 & 19 & 1,7 \\
\hline $\begin{array}{l}\text { 6- Travailleurs de la } \\
\text { production }\end{array}$ & 343 & 77,6 & 369 & 67,6 & 51 & 47,7 & 763 & 69,7 \\
\hline 7- Indéterminés & 0 & - & 1 & 0,2 & 2 & 1,9 & 3 & 0,3 \\
\hline TOTAL & 442 & 100 & 546 & 100 & 107 & 100 & 1095 & 100 \\
\hline
\end{tabular}

Source: Registres de publications de bans, paroisse Ste-Brigide, 1905-1914. 
TABLEAU 2

Origine géographique des époux et épouses unis à Sainte-Brigide, 1908-1914: répartition globale.

\begin{tabular}{|c|c|c|c|c|c|c|c|c|c|c|}
\hline & Nombre & & $\begin{array}{c}\text { Bapt. à } \\
\text { Montréal }\end{array}$ & $\%$ & $\begin{array}{c}\text { Bapt. dans } \\
\text { une autre ville }\end{array}$ & $\%$ & Ensemble & $\%$ & $\begin{array}{l}\text { Bapt. } \\
\text { à la campagne }\end{array}$ & $\%$ \\
\hline $\begin{array}{l}\text { SECTION A } \\
\text { Résidant à Ste-Brigide } \\
\text { au moment du mariage }\end{array}$ & $\begin{array}{l}\text { Hommes et } \\
\text { Femmes } \\
\text { Femmes } \\
\text { Hommes }\end{array}$ & $\begin{array}{l}932 \\
651 \\
281\end{array}$ & $\begin{array}{r}354 \\
260 \\
94\end{array}$ & $\begin{array}{l}38,0 \\
39,9 \\
33,5\end{array}$ & $\begin{array}{r}239 \\
173 \\
66\end{array}$ & $\begin{array}{l}25,6 \\
26,6 \\
23,5\end{array}$ & $\begin{array}{l}593 \\
433 \\
160\end{array}$ & $\begin{array}{l}63,6 \\
66,5 \\
57,0\end{array}$ & $\begin{array}{l}339 \\
218 \\
121\end{array}$ & $\begin{array}{l}36,4 \\
33,5 \\
43,0\end{array}$ \\
\hline $\begin{array}{l}\text { SECTION B } \\
\text { Résidant à Montréal } \\
\text { sauf SB au moment du } \\
\text { mariage }\end{array}$ & $\begin{array}{l}\text { Hommes et } \\
\text { Femmes } \\
\text { Femmes } \\
\text { Hommes }\end{array}$ & $\begin{array}{r}354 \\
21 \\
333\end{array}$ & $\begin{array}{r}136 \\
9 \\
127\end{array}$ & $\begin{array}{l}38,4 \\
42,9 \\
38,1\end{array}$ & $\begin{array}{r}76 \\
6 \\
70\end{array}$ & $\begin{array}{l}21,5 \\
28,6 \\
21,0\end{array}$ & $\begin{array}{r}212 \\
15 \\
197\end{array}$ & $\begin{array}{l}59,9 \\
71,5 \\
59,1\end{array}$ & $\begin{array}{r}142 \\
6 \\
136\end{array}$ & $\begin{array}{l}40,1 \\
28,5 \\
40,9\end{array}$ \\
\hline $\begin{array}{l}\text { SECTION A + B } \\
\text { Résidant à Montréal y } \\
\text { compris à SB au } \\
\text { moment du mariage }\end{array}$ & $\begin{array}{l}\text { Hommes et } \\
\text { Femmes } \\
\text { Femmes } \\
\text { Hommes }\end{array}$ & $\begin{array}{r}1286 \\
672 \\
614\end{array}$ & $\begin{array}{l}490 \\
269 \\
221\end{array}$ & $\begin{array}{l}38,1 \\
40,0 \\
36,0\end{array}$ & $\begin{array}{l}315 \\
179 \\
136\end{array}$ & $\begin{array}{l}24,5 \\
26,7 \\
22,1\end{array}$ & $\begin{array}{l}805 \\
448 \\
357\end{array}$ & $\begin{array}{l}62,6 \\
66,7 \\
58,1\end{array}$ & $\begin{array}{l}481 \\
224 \\
257\end{array}$ & $\begin{array}{l}37,4 \\
33,3 \\
41,9\end{array}$ \\
\hline $\begin{array}{l}\text { SECTION C } \\
\text { Résidant hors de } \\
\text { Montréal au moment du } \\
\text { mariage }\end{array}$ & $\begin{array}{l}\text { Hommes et } \\
\text { Femmes } \\
\text { Femmes } \\
\text { Hommes }\end{array}$ & $\begin{array}{r}72 \\
7 \\
65\end{array}$ & $\begin{array}{l}8 \\
1 \\
7\end{array}$ & $\begin{array}{l}11,1 \\
14,3 \\
10,8\end{array}$ & $\begin{array}{r}13 \\
1 \\
12\end{array}$ & $\begin{array}{l}18,1 \\
14,3 \\
18,4\end{array}$ & $\begin{array}{r}21 \\
2 \\
19\end{array}$ & $\begin{array}{l}29,2 \\
28,6 \\
29,2\end{array}$ & $\begin{array}{r}51 \\
5 \\
46\end{array}$ & $\begin{array}{l}70,8 \\
71,4 \\
70,8\end{array}$ \\
\hline Total & $\begin{array}{c}\text { Hommes et } \\
\text { Femmes }\end{array}$ & 1358 & 498 & 36,7 & 328 & 24,1 & 826 & 60,8 & 532 & 39,2 \\
\hline
\end{tabular}

Source: Registres de publications de bans, paroisse Ste-Brigide, 1908-1914 
alimentent aussi régulièrement. Dans ces cas, ce sont des paroisses urbaines et ouvrières de l'Immaculée-Conception à Trois-Rivières, de Sait-Roch et de Saint-Sauveur à Québec qu'on émigre le plus vers SainteBrigide. Cependant, presque tous les comtés du Québec, des plus voisins au plus lointains, envoient des leurs à Montréal et à Sainte-Brigide.

Cette prépondérance de l'immigration québécoise et trifluvienne dans la paroisse, jointe au fait que les banlieues industrielles de Montréal fournissent également de forts effectifs à Sainte-Brigide, conduit à penser qu'au début du 20e siècle l'espace industriel extérieur alimente grandement l'espace industriel paroissial. Car, pour la période, près de $61 \%$ des jeunes mariés et mariées de Sainte-Brigide proviennent de régions déjà urbanisées et industrialisées. Dès les années 1880-1890, donc, la paroisse Sainte-Brigide, en plein coeur du quartier SainteMarie, doit sa croissance démographique essentiellement à deux facteurs: l'accroissement naturel interne et l'arrivée de gens venus d'autres régions industrialisées de l'île de Montréal et du Québec. Nos jeunes époux des années $1908-1914^{8}$ ont donc derrière eux une tradition courte sans doute, mais déjà ancrée de vie urbaine et industrielle.

L'analyse de l'hérédité du statut social ${ }^{9}$ des travailleurs et des travailleuses unis à Sainte-Brigide entre 1905 et 1914 permet de documenter davantage cette affirmation. Chez les hommes, pour lesquels les données sont plus sûres parce que plus complètes, on observe que plus de huit travailleurs sur dix vivant à Sainte-Brigide, ou ailleurs à Montréal au moment de leur mariage, ont un père lui-même travailleur. L'hérédité du statut social l'emporte largement même chez ceux qui habitent hors de Montréal, dans des plus petits centres, voire dans des villages, où déjà six travailleurs sur dix ont un père travailleur lui aussi. Cette hérédité du statut social très fortement significative, même chez les femmes, malgré la relative fragilité des données, contribue à son tour à préciser le portrait qui se dégage de plus en plus nettement: au début du $20 \mathrm{e}$ siècle, les travailleurs brigidains tout comme les travailleurs montréalais, forment un groupe qui se reproduit par lui-même. Yves Lequin parle d'endogénie sociale à ce propos dans un article sur les ouvriers de la région lyonnaise ${ }^{10}$. On pourrait reprendre l'expression

8 Ce n'est qu'à partir de 1908 que le lieu et la date du baptême sont indiqués.

9 La détermination de l'hérédité du statut social s'est présentée simplement pour les garçons; en effet la profession des mariés et de leurs pères étant presque toujours mentionnée, il était facile de trancher la question. Pour les filles au contraire, des choix plus arbitraires se sont imposés du fait que leur profession n'est jamais indiquée. On a alors considéré comme travailleuses toutes les filles dont le père était lui-même travailleur, de même que toutes celles qui résidaient à Montréal et dont le père était cultivateur. Cette logique du bon sens ne peut se targuer d'une rigueur scientifique irréprochable. Mais agir autrement signifiait abandonner toute velléité d'analyse de l'hérédité et de l'endogamie sociales des travailleuses, ce à quoi je n'ai pu me résoudre, convaincue que la fragilité des données, valables seulement comme indices, est préférable au silence total.

${ }_{10}$ Yves Lequin, «La formation du prolétariat industriel dans la région lyonnaise au 19e siècle: approches méthodologiques et premiers résultats», in Le Mouvement Social, 97 (octobredécembre 1976): 121-137. 
pour les travailleurs et les travailleuses mariés à Sainte-Brigide entre 1905 et 1914.

Cette hérédité du statut social et l'endogénie sociale remarquées constituent les premiers caractères d'une cohésion sociale qui trouve l'une de ses manifestations les plus révélatrices lorsque vient pour chacun et chacune le moment de choisir son conjoint. On s'aperçoit alors qu'à l'endogénie sociale s'ajoute le plus souvent sinon presque toujours l'endogamie sociale. 92\% des femmes choisissent mari dans leur classe d'origine. A peine plus de six Brigidaines sur cent accèdent par le

TABLEAU 3

L'hérédité du statut social chez les travailleurs mariés dans Ste-Brigide: 1905-1914.

\begin{tabular}{|l|c|c|c|c|c|c|}
\hline \multicolumn{1}{|c|}{$\begin{array}{c}\text { Statut } \\
\text { social } \\
\text { du père }\end{array}$} & $\begin{array}{c}\text { Trav. } \\
\text { résident } \\
\text { à Ste-Brigide }\end{array}$ & $\%$ & $\begin{array}{c}\text { Trav. } \\
\text { résident } \\
\text { à Mtl - SB }\end{array}$ & $\%$ & $\begin{array}{c}\text { Trav. } \\
\text { résident } \\
\text { hors de Mtl }\end{array}$ & $\%$ \\
\hline Travailleur & 135 & 79,4 & 201 & 83,4 & 17 & 58,6 \\
\hline Cultivateur & 20 & 11,8 & 25 & 10,4 & 8 & 27,6 \\
\hline $\begin{array}{l}\text { Autre } \\
\text { surtout } \\
\text { commerce et } \\
\text { prof. libérale }\end{array}$ & 15 & 8,8 & 15 & 6,2 & 4 & 13,8 \\
\hline Total & 170 & 100 & 241 & 100 & 29 & 100 \\
\hline
\end{tabular}

Source: Registres de publications de bans, paroisse Sainte-Brigide, 1905-1914

TABLEAU 4

L'hérédité du statut social chez les travailleuses mariées dans Ste-Brigide: 1905-1914

\begin{tabular}{|l|c|c|c|c|c|c|}
\hline \multicolumn{1}{|c|}{$\begin{array}{c}\text { Statut } \\
\text { social du } \\
\text { père }\end{array}$} & $\begin{array}{c}\text { Trav. } \\
\text { rés. à SB }\end{array}$ & $\%$ & $\begin{array}{c}\text { Rés. } \\
\text { à Mtl - SB }\end{array}$ & $\%$ & $\begin{array}{c}\text { Rés. } \\
\text { hors Mtl }\end{array}$ & $\%$ \\
\hline Travailleur & 395 & 91,2 & 17 & 85 & 9 & 69,2 \\
\hline Cultivateur & 38 & 8,8 & 3 & 15 & 4 & 30,8 \\
\hline $\begin{array}{l}\text { Autre } \\
\text { surtout } \\
\text { commerce et } \\
\text { prof. libérale }\end{array}$ & 0 & 0 & 0 & 0 & 0 & 0 \\
\hline Total & 433 & 100 & 20 & 100 & 13 & 100 \\
\hline
\end{tabular}

Source: Registres de publications de bans, paroisse Sainte-Brigide, 1905-1914 
TABLEAU 5

L'endogamie sociale des travailleurs mariés à Sainte-Brigide: 1905-1914

\begin{tabular}{|l|c|c|c|c|c|c|}
\hline \multicolumn{1}{|c|}{$\begin{array}{c}\text { Statut } \\
\text { social du } \\
\text { beau-père }\end{array}$} & $\begin{array}{c}\text { Trav. } \\
\text { résidant } \\
\text { à Ste-Brigide }\end{array}$ & $\%$ & $\begin{array}{c}\text { Trav. } \\
\text { résidant à } \\
\text { Mtl - SB }\end{array}$ & $\%$ & $\begin{array}{c}\text { Trav. rés. } \\
\text { hors de Mtl }\end{array}$ & $\%$ \\
\hline Travailleur & 151 & 81,2 & 207 & 84,8 & 19 & 86,4 \\
\hline Cultivateur & 17 & 9,1 & 21 & 8,6 & 2 & 9,1 \\
\hline $\begin{array}{l}\text { Autre } \\
\text { surtout } \\
\text { commerce et } \\
\text { prof. libérale }\end{array}$ & 18 & 9,7 & 16 & 6,6 & 1 & 4,5 \\
\hline Total & 186 & 100 & 244 & 100 & 22 & 100 \\
\hline
\end{tabular}

Source: Registres de publications de bans, paroisse Sainte-Brigide, 1905-1914

TABLEAU 6

L'endogamie sociale des travailleuses mariées à Sainte-Brigide: 1905-1914

\begin{tabular}{|l|c|c|c|c|c|c|}
\hline \multicolumn{1}{|c|}{$\begin{array}{c}\text { Statut } \\
\text { social } \\
\text { du marié }\end{array}$} & $\begin{array}{c}\text { Trav. } \\
\text { résidente } \\
\text { à Ste-Brigide }\end{array}$ & $\%$ & $\begin{array}{c}\text { Trav. rés. à } \\
\text { Mtl - SB }\end{array}$ & $\%$ & $\begin{array}{c}\text { Trav. } \\
\text { résidente } \\
\text { hors de Mtl }\end{array}$ & $\%$ \\
\hline Travailleur & 385 & 91,7 & 20 & 100 & 11 & 91,7 \\
\hline Cultivateur & 9 & 2,1 & 0 & 0 & 0 & 0 \\
\hline $\begin{array}{l}\text { Autre } \\
\text { surtout } \\
\begin{array}{l}\text { commerce et } \\
\text { prof. libérales }\end{array}\end{array}$ & 26 & 6,2 & 0 & 0 & 1 & 8,3 \\
\hline Total & 420 & 100 & 20 & 100 & 12 & 100 \\
\hline
\end{tabular}

Source: Registres de publications de bans, paroisse Sainte-Brigide, 1905-1914

mariage à un statut social supérieur. Les travailleurs aussi trouvent leur compagne dans leur classe, dans au moins huit cas sur dix. Ils éprouvent par ailleurs des difficultés comparables à celles des femmes à se marier au-dessus de leur condition: un travailleur de Sainte-Brigide sur dix épouse une fille de commerçant ou de professionnel.

A cette cohésion sociale s'ajoute enfin la cohésion ethnique. En effet, les mariages inter-ethniques à Sainte-Brigide représentent moins de $8 \%$ de toutes les unions célébrées dans la paroisse entre 1905 et 1914. Le taux d'homogénéité ethnique grimpe même à tout près de $96 \%(95,8 \%)$ si l'on ne considère que les noces célébrées entre francophones, qu'ils soient canadiens ou américains. 
Dans une paroisse où $80 \%$ de la population est ouvrière, le monde des travailleurs brigidains se caractérise par une forte cohésion sociale aux traits clairement dessinés: on naît le plus souvent en ville, dans la classe laborieuse, et on y prend mari ou femme; dans le cas où le jeune homme exerce un métier réclamant une certaine qualification, comme ceux de menuisier, plombier ou tailleur, une fois sur quatre il perpétue le métier de son père et parfois même pratique celui de son beau-père ${ }^{11}$. Par ailleurs, la cohésion ethnique se conjugue à la cohésion sociale. Les conditions sont toutes réunies pour assurer toujours davantage la reproduction de la classe laborieuse à partir de ses propres effectifs. Sainte-Brigide offre donc un terrain d'observation privilégié pour l'analyse du cadre de vie familiale et du mariage en milieu ouvrier canadienfrançais.

\section{2 - Le cadre de la vie familiale}

Tous les historiens des conditions de vie ouvrière, tant au Québec qu'à l'étranger ${ }^{12}$, ainsi qu'une pléiade d'écrivains dont Zola reste le plus célèbre, ont souligné la difficulté de concilier au 19e siècle et au début du 20e, travail industriel et vie familiale. Cette difficulté tient bien sûr essentiellement aux conditions de travail des ouvriers. L'embauche précoce des enfants, le travail à l'extérieur des femmes mariées, du moins pendant certaines périodes de leur vie, les longues heures imposées et l'épuisement qui en résulte, la diversité des horaires, la mobilité d'une main-d'oeuvre qui suit les possibilités d'emploi, ainsi que la mort trop souvent prématurée sont autant de facteurs qui rendent compte d'un modèle d'organisation familiale élargie et souple, apte à compenser, par les formes de solidarité que les membres développent, l'incertitude de l'emploi, les déficiences du salaire et celles du soutien public ou privé.

Les travailleurs brigidains évoluent dans un cadre familial différent de celui des élites bourgeoises à cause des conditions de vie et de travail auxquelles ils sont assujettis. Toutefois les sources n'ont révélé de leur part ni contestation ni refus du modèle d'organisation familiale proposé par la bourgeoisie ou le clergé. Celui-ci cependant, dépassé par l'ampleur du mouvement de déstructuration familiale apparente en milieu ouvrier, insiste sur la valorisation de la famille. Les responsables diocésains accordent volontiers les chartes de constitution des congrégations laïques dans les paroisses; les curés exhortent les jeunes à fon-

11 Registres de publications de bans, paroisse Sainte-Brigide, 1905-1914.

12 Plusieurs historiens de la condition ouvrière se sont intéressés à la famille. Voir en particulier, parmi les travaux récents, ceux de Tamara K. Hareven, Family Time and Industrial Time (Cambridge, Cambridge University Press, 1982), 474 p.; de Jean-Pierre Navailles, La famille ouvrière dans l'Angleterre victorienne (Éditions du Champ Vallon, 1983), 334 p.; et, pour ce qui regarde plus spécifiquement la situation montréalaise, ceux de Bettina Bradbury, «The Family Economy and Work in an Industrializing City: Montreal in the 1870's», in Société historique du Canada/Communications historiques, (1979): 71-96, et les articles écrits depuis. 
der des familles chrétiennes; leurs vicaires organisent des loisirs familiaux; les formes d'encouragement à la vie familiale sont très nombreuses dans les paroisses ouvrières.

L'analyse des cahiers de prônes et des registres de publications de bans livre, de façon indirecte, plusieurs renseignements sur le cadre de la vie familiale des travailleurs brigidains. Ils suppléent en partie à l'absence de témoignages directs et de descriptions contemporaines de la vie familiale en milieu ouvrier montréalais.

Ni contestation, ni refus du modèle familial proposé par le clergé... Quoique sans être absolument formels, les indices convergent en ce sens.

Tout d'abord le nombre impressionnant des Enfants de Marie. Car si à peine huit ouvriers sur cent appartiennent à la congrégation des Jeunes Gens au moment de leur mariage, contre près de $16 \%$ des jeunes professionnels ou fils de notables, en revanche $36 \%$ des épousées sont Enfants de Marie, autant que dans les classes plus aisées. Or les congrégations sont l'un des moyens dont s'est doté le clergé pour encadrer la population paroissiale. Le message clérical rejoint donc davantage de filles que de garçons. Ce succès relativement plus important auprès des jeunes filles s'explique-t-il par la publicité scolaire à ce propos, par l'encouragement plus grand donné aux filles au sein même de la famille? Les sources ne permettent pas de répondre, mais il reste certain que les conséquences culturelles en ont été nombreuses, entre autres en assurant une plus grande intégration chez les jeunes filles du modèle familial proposé par l'Église et peut-être une volonté plus forte chez elle de le réaliser lorsqu'elles seront devenues à leur tour les piliers de la famille.

Marie-Louise Moussette (47 ans) veuve de Pierre Picotte, demeure à 859 Demont(igny) chez le veuf Hormisdas Daoust où elle est censée tenir maison depuis mai 1913. Daoust devait l'épouser en avril, ou mai, ou juin... Les costumes de noces étaient faits... cependant on ne voit rien venir... et la femme reste avec Daoust, qui a trois enfants: $12,10,4$ ans... ${ }^{13}$

Les témoignages de ce genre sont aussi imagés qu'exceptionnels. Ils ne reviennent que neuf fois sur 1105 mariages et concernent ceux qui ont choisi de régulariser leur situation. Si l'on ignore le nombre de couples brigidains qui vivent en concủbinage, on peut cependant se donner une idée approximative de la faveur de cette pratique en estimant le taux de naissances illégitimes.

Entre 1880 et 1914, cette proportion reste infime ${ }^{14}$. Le taux moyen s'établit à $1,4 \%$ entre 1880 et 1899 et à $1,1 \%$ entre 1900 et 1914 . C'est

13 Cahiers de prônes, paroisse Sainte-Brigide, 6 juillet 1913.

14 Registres de baptêmes, paroisse Sainte-Brigide, 1880-1914. 
très peu. Il est vrai toutefois qu'à Montréal beaucoup de célibataires abandonnaient leur enfant chez les Soeurs Grises ou les Soeurs de la Miséricorde avant le baptême, ce qui explique en partie le sous-enregistrement des naissances illégitimes dans la paroisse même. D'après quelques auteurs français, le taux d'illégitimité oscille autour de 15 à $20 \%$ dans la deuxième moitié du $19 \mathrm{e}$ siècle dans les régions industrialisées de la France ${ }^{15}$. A côté de tels chiffres, le concubinage et les naissances illégitimes à Sainte-Brigide prennent figure de cas exceptionnels.

Mais si la majorité des couples d'ouvriers se marient, les circonstances de leur vie de travail et de pauvreté infléchissent la destinée des familles.

En effet, d'après les registres de publications de bans, les familles restreintes des travailleurs mariés dans la paroisse se caractérisent, si tant est qu'on puisse généraliser, par l'absence des parents des mariés et par l'éparpillement des jeunes à partir de l'adolescence. Au moment du mariage, près de $45 \%$ des couples de parents sont déjà défaits ${ }^{16}$; la mort répond presque toujours de ces séparations, un parent ou même les deux étant déjà décédés le jour des noces de leurs enfants. Cependant il faut souligner les dix-huit cas de séparation dûs au départ volontaire du père, depuis plus de dix ans dans la majorité des cas connus, même si ce phénomène reste marginal.

Ainsi presqu'un couple de parents sur deux n'existe plus le jour du mariage de ses enfants. En conséquence, les rejetons nés de ces nouvelles unions ont peu de chances de connaître et de fréquenter leurs grands-parents, surtout s'ils résident dans une autre paroisse, comme c'est souvent le cas. De même les jeunes conjoints des années 19051914 n'ont pas dû, toujours à cause de la faible espérance de vie, voir très souvent leurs propres grands-parents.

De plus, même lorsque leurs parents vivent, ou au moins un des deux, les jeunes ont tendance à quitter le foyer familial avant leur mariage. C'est du moins ce que montre l'étude des lieux de résidence respectifs des conjoints et de leurs parents.

Pour obtenir le nombre de personnes qui se présentent pour la première fois devant l'autel entre 1905 et 1914, à Sainte-Brigide, les veufs et les veuves qu'on suppose ne plus vivre chez leurs parents au moment de leur remariage ont été éliminés de nos calculs. Il reste 963

\footnotetext{
15 Louis Chevalier, Classes laborieuses et classes dangereuses à Paris, pendant la première moitié du 19e siècle (Paris, Plon, 1958), 385. Georges Duveau, La vie ouvrière en France sous le Second Empire (Paris, Gallimard, 1946), 425-428. Pierre Pierrard, La vie ouvrière à Lille sous le Second Empire (Paris, Bloud et Guay, 1968), 118.

${ }_{16}$ Les archives de Sainte-Brigide ont conservé les publications de bans des 1105 couples de travailleurs entre 1905 et 1914. On a des informations sur 1993 des 2210 couples de parents des mariés ainsi obtenus, c'est-à-dire sur 90,2\% de l'ensemble.
} 
hommes et 1018 femmes dont, 440 hommes et 336 femmes ne résidant plus chez leurs parents au moment des noces, soit respectivement $45,7 \%$ et $33 \%$ du total: c'est donc tout près d'un jeune homme sur deux et une jeune femme sur trois. L'écart des taux entre les sexes s'explique sûrement en partie par la faiblesse des salaires féminins qui ne permettent pas aux jeunes filles de quitter leur famille, car la pension risque d'être plus élevée ailleurs que chez leurs parents; en partie aussi par la plus grande jeunesse des femmes au mariage, point sur lequel on aura l'occasion de revenir; et en partie certainement par les services qu'elles rendent dans la maison jusqu'à leurs noces.

Quant à ceux qui s'éloignent du foyer parental, où vont-ils demeurer? «Chez Jos. Lachance», «chez dame Mousseau», de telles mentions reviennent souvent sous la plume du curé de la paroisse, $\mathbf{M}$. Demers. En effet, une bonne partie de ces jeunes vont habiter dans d'autres familles, où ils pensionnent. Sur les 1530 adresses connues pour les conjoints des deux sexes (69,2\% de l'ensemble des adresses), 65 cas de pensionnaires sont spécifiquement identifiés: cela représente un petit 4,3\%. Cependant, il est évident que le nombre réel de pensionnaires dépasse de beaucoup ce pourcentage; le curé n'a tout simplement pas inscrit chaque fois le fait. Si l'on considère par ailleurs comme pensionnaires plutôt que comme concubins les 94 promis qui vivent deux par deux à la même adresse ${ }^{17}$ et si on leur ajoute tous ceux qui sont établis depuis moins d'un an dans la paroisse, on obtient un total de 220 pensionnaires, soit $14,4 \%$ du total. Mais cela sous-estime certainement encore de beaucoup la réalité, surtout si l'on tient compte du nombre de jeunes qui ont quitté leurs parents avant leur mariage.

Ils ont quitté leurs parents, mais pas nécessairement leur famille. En effet, c'est souvent par le biais de la pension que l'on prend contact avec cette solidarité familiale élargie qui caractérise les travailleurs brigidains. Non que la pension soit sa seule forme d'expression, mais les registres de publications de bans se taisent sur ses autres manifestations. Combien de fois voit-on des mentions comme celle-ci: «Depuis $31 / 2$ mois dans Ste-Brigide chez son beau-frère», ou comme celle-ci: «chez sa tante, dame J.-B. Lamontagne» ou encore comme celle-ci: «chez son frère André Blanchet»? Autant de signes de cette entraide familiale élargie. Or, dans cette famille élargie, on n'inclut pas seulement les membres rattachés par les liens du sang; mais aussi les pensionnaires étrangers qui s'installent dans des familles déjà constituées. Cette habitude d'offrir pension constitue une forme de sociabilité particulière. En plus des avantages matériels réciproques qu'ils tirent de ce système, la cohabitation permet, entre la famille hôte et le ou la pensionnaire la circulation d'informations, l'échange des expériences et aussi une meil-

\footnotetext{
17 On ne les reconnaît pas comme concubins parce qu'ils ne sont pas jugés comme tels explicitement par le curé Demers.
} 
leure connaissance, du moins intellectuelle, des divers coins du Québec ou d'ailleurs d'où tous originent. En ce sens, la cohabitation favorise l'accroissement du savoir des travailleurs.

Par ailleurs, des liens se tissent qui conduisent plus d'une fois à l'autel. Il arrive que les futurs conjoints se rencontrent lorsque l'un des deux pensionne dans la famille de l'autre; directement chez ses parents dans sept cas (et fort probablement dans six autres cas) ou chez l'un de ses frères ou soeurs (deux cas). En outre, comme certaines familles accueillent plusieurs hôtes, 94 des futurs conjoints se sont connus à l'époque où ils pensionnent au même endroit. Sur 220 pensionnaires, entre 103 et 109 ont donc connu leur conjoint lors de la cohabitation, ce qui représente entre $46,8 \%$ et $49,5 \%$ du total. Ils sont les cas limite d'une tendance généralisée à choisir son ou sa partenaire dans un rayon géographique très restreint autour de son propre lieu de résidence. En effet, plus du tiers $(34,6 \%)$ des bans de mariages publiés à SainteBrigide proposent d'unir deux paroissiens brigidains et un autre quart $(25,1 \%)$ promet l'un à l'autre une résidente de la paroisse et un résident d'une paroisse adjacente: Saint-Pierre-Apôtre, Sainte-Catherined'Alexandrie, Sacré-Coeur-de-Jésus, Saint-Vincent-de-Paul ou St.Mary's. Au total, 59,7\% des mariages se forment entre gens habitant très près l'un de l'autre et $87,6 \%$ unissent deux Montréalais. Quant on s'intéresse maintenant aux couples formés de deux Brigidains, on note qu'outre les 108 à 120 personnes logées à la même adresse et dont on a parlé plus haut ${ }^{18}, 108$ autres paroissiens habitent sur la même rue avant leur mariage à moins de dix maisons de distance. Tout près de $60 \%$ des promis résident donc vraiment très près les uns des autres. Mais ces chiffres sous-estiment la réalité de deux façons: d'une part parce que, malgré les atlas des compagnies d'assurances, on n'a pu évaluer le nombre de ceux qui habitent le même pâté de maisons; et puis parce que l'on ne connaît l'adresse exacte des deux partenaires que dans 239 cas sur 374 .

Tous ces chiffres traduisent la vigueur et l'intensité des liens de voisinage des travailleurs brigidains. De tels pourcentages compensent en partie l'absence de tout témoignage de leur sociabilité domestique et paroissiale. Ils permettent d'imaginer des maisons facilement ouvertes aux voisins qui sont aussi les amis, les conversations de balcons, de perron ou d'arrière-cour, une vie de rue bien entretenue, surtout durant l'été, et des contacts faciles entre les paroissiens, en dépit d'une mobilité très grande, difficile cependant à mesurer précisément, mais que révèlent quelques notes du curé Demers: «Eugénie Gagnon: le 30 octobre à S.Pierre; le 6 novembre au Sacré-Coeur; le 19 novembre à St-Vincent-

\footnotetext{
1894 promis et entre 14 et 26 autres qui se sont connus parce qu'ils habitaient sous le toît d'un parent d'un des conjoints.
} 
de-Paul.» ${ }^{19}$ «Le garçon pensionne depuis 3 mois à 101, Hôtel-de-Ville. A Montréal depuis un an et demi. Aux Etats-Unis pendant deux ans. $\gg^{20}$ «Blanche Boyer: A St-Jacques depuis deux mois... n'a pas de domicile fixe.» ${ }^{21}$

Ces mentions, comme les autres données utilisées ici, dévoilent un phénomène mais interdisent en même temps, par leur rareté ou leur imprécision, d'en prendre la mesure exacte. C'est pourquoi, à la fin de cette section sur le cadre de la vie familiale des travailleurs brigidains, on obtient davantage une eau-forte, aux traits et aux teintes parfois voilés ou un peu flous, que le tableau incisif et bien net qu'on aurait voulu dégager. Une famille restreinte souvent éparpillée, une solidarité familiale élargie, une sociabilité domestique et une vie de quartier intense, une mobilité individuelle et familiale sinon généralisée, du moins très fréquente, et pour tout dire une intégration du modèle catholique de la famille, telles semblent être les caractéristiques de la famille ouvrière brigidaine au début du siècle ${ }^{22}$.

\section{3 - La cérémonie nuptiale}

On l'a vu, la grande majorité des couples se marient. Et pourtant, quelle difficulté de savoir comment les noces étaient vécues en milieu ouvrier! Rien sur les préparatifs, rien sur les réjouissances, sinon cette remarque du curé Demers: «Jour du mariage... riz... confettis... vieilles bottines... manches de fouets... bâtons noueux... en prévision des râclées futures!» ${ }^{23}$

Près de vingt-cinq ans pour les hommes, vingt-deux ans pour les femmes, c'est l'âge moyen des travailleurs unis pour la première fois entre 1905 et 1914 à Sainte-Brigide. Ce qui laisse supposer qu'on attend d'avoir amassé un petit pécule, acquis au long des années déjà passées sur le marché du travail, avant de se présenter devant l'autel. Mais ces moyennes cachent des variations d'âge importantes puisque près d'un garçon sur sept $(13,3 \%)$ et plus d'une fille sur trois $(35,2 \%)$ sont encore mineurs le jour des noces.

La cérémonie est placée sous le signe des commencements. En effet, on se marie de préférence au début de la journée, au début de la semaine et au printemps. Comme le choix du moment du mariage est un révélateur privilégié du comportement culturel, il serait tout particulièrement intéressant de comparer les habitudes urbaines aux tradi-

19 Registres de publications de bans, paroisse Sainte-Brigide, 25 novembre 1905.

20 Idem, 27 novembre 1905.

21 Idem, 6 octobre 1908.

22 L'usage de louer une chambre à des pensionnaires étrangers ou à un membre de la famille est également répandu dans les quartiers ouvriers de Sainte-Anne et de Saint-Jacques à Montréal. B. Bradbury, loc. cit.

${ }^{23}$ Cahiers de prônes, paroisse Sainte-Brigide, 12 novembre 1911. 
tions rurales; mais la documentation est rare sur cette question, aussi bien pour les quartiers urbains que pour les campagnes.

«Heures des mariages: être régulier, surtout quand plusieurs mariages sont célébrés à la même heure.» ${ }^{24}$ Dans le comté de Bellechasse, on se mariait, au début du siècle, vers huit ou neuf heures ${ }^{25}$. Seuls les veufs et les veuves convolaient plus matinalement afin d'éviter les quolibets qui, semble-t-il, pleuvaient sur eux. A Sainte-Brigide, entre 1905 et 1914, ils conservent cette habitude de se remarier au lever du jour et plus d'une union consacrée dès cinq heures les concerne, peut-être parce que la raillerie continue de fuser, ainsi qu'en témoigne cette réflexion du curé Demers lui-même:

Francis Presseau est pied bot. 72 ans. Marie Durocher est valide du côté gauche mais paralysée du côté droit. 59 ans. Le témoin, Camille Marsolais est orné d'une jambe de bois et armé d'une canne en merisier pour étayer sa gauche. Dans le cas où surviendraient des enfants, on s'attend à en voir arriver un avec un oeil en vers (sic), des cheveux en filasse, une patte de piano... la canne à la main. ${ }^{26}$

Cependant, les célibataires ne tardent pas non plus. Pour toute la période, le curé bénit un minimum des deux-tiers des mariages à 6:30 heures ou avant, quatre sur cinq en 1907, et ce, même s'il offre des cérémonies jusqu'à 7:30 heures. Les mariages du soir restent très rares voire secrets, on n'en a pas publié les bans; ce sont des mariages «pressés», comme on dit, ceux des femmes trop visiblement enceintes.

Par ailleurs, à peine $8 \%$ des époux choisissent de célébrer le mariage le samedi, mais la moitié d'entre eux $(48,2 \%)$ s'unissent le lundi et un autre tiers $(34,3 \%)$ le mardi. On peut donc imaginer une cérémonie courte, bâclée en une demi-heure pour faire place aux suivants, unissant maintes fois plusieurs couples en même temps et dont la simplicité est proportionnelle au prix acquitté. Précède-t-elle le labeur quotidien, qu'elle n'entrave pas tant elle est matinale ou, pour l'occasion, désertet-on l'atelier? La documentation se tait sur cette question.

Les mariages des femmes enceintes sont les seuls pour lesquels on passe outre la prohibition du Carême et de l'Avent. Habituellement, les conjoints préfèrent les mois du printemps et de l'automne. Mai et juin sont les plus recherchés: à eux seuls ils rassemblent plus du quart $(26,9 \%)$ des unions; octobre et novembre suivent avec près d'un autre quart $(22,9 \%)$. Les six mois restants se partagent l'autre moitié des mariages, avec une légère préférence pour août et septembre. Il serait intéressant

\footnotetext{
24 Cahiers de prônes, paroisse Sainte-Brigide, 26 mai 1907. 1981.

Je tiens cette information du père Benoît Lacroix, qui m'a accordé un entretien en juillet

26 Registres de publications de bans, paroisse Sainte-Brigide, 14 septembre 1914. Les promis sont tous deux veufs.
} 
de pouvoir comparer avec la pratique en milieu rural, où il semble que les mêmes mois soient choisis, afin de préciser dans quelle mesure les traditions sont conservées à la ville.

Le règlement du prix de la cérémonie est toujours à la charge du jeune homme, qui y consacre une grosse fraction, sinon la totalité de son salaire moyen d'une semaine ${ }^{27}$. En effet, le mariage ouvrier le plus courant est à cinq dollars ou moins (dans $57,3 \%$ des cas). Pour un extra, un tiers $(32,0 \%)$ des ouvriers sont prêts à payer jusqu'à dix dollars, mais moins d'un dixième peut s'offrir davantage. Par comparaison, les filles et les fils de notables brigidains se marient rarement à moins de quinze dollars: les deux-tiers consentent au moins cette somme. Certains, plus modestes quand même, se contentent d'un mariage à dix dollars, mais à peine moins du cinquième des conjoints de cette catégorie $(16,7 \%)$ osent mettre moins.

On ignore ce que l'Église offre en échange de ces deniers, toujours versés d'avance. Mais s'il faut en croire les quelques indications en marge des publications de bans, les cloches, et l'orgue, de même que la lumière électrique ou le tapis constituent toujours un supplément, même dans les mariages à dix dollars. A titre d'exemple, voici les échos de l'union la plus onéreuse célébrée durant la décade dans la paroisse, celle d'un manufacturier et d'une fille d'un bourgeois brigidain. La cérémonie vaut vingt-cinq dollars, ce qui signifie qu'elle a été célébrée au centre de l'église, au grand autel. A cette somme, on a ajouté vingtcinq dollars de lumière électrique, cinq dollars d'orgue et cinq dollars de chant, pour un total de soixante dollars ${ }^{28}$. On imagine sans peine la brillance de ce mariage par rapport à ceux de deux dollars cinquante ou même de cinq dollars qui sont le lot des travailleurs. Mais l'évocation doit s'arrêter là, faute de documents pour l'alimenter.

Quant au mariage des congréganistes, il présente un peu plus d'apparât. En effet, il semble que, quel que soit le prix de la cérémonie, il comprenne automatiquement le choeur de chant de la congrégation, celui des Enfants de Marie ou celui des Jeunes Gens selon le cas. Si le marié appartient à la congrégation des Jeunes Gens, il peut, à son gré, porter une bandoulière; si l'épouse est Enfant de Marie, elle est coiffée d'une couronne. Ces deux distinctions sont également gratuites. Par ailleurs, les congréganistes bénéficient de réductions sur les autres suppléments offerts par l'Église aux mariés. Par exemple, les fauteuils coûtent un dollar au lieu de cinq chacun, l'orgue résonne pour deux dollars plutôt que pour cinq, on consent des rabais sur les prix du tapis,

27 L'historiographie québécoise donne beaucoup d'informations sur les salaires des travailleurs montréalais à cette époque. Voir, entre autres, Herbert Brown Ames, The City Below the Hill (Toronto, University of Toronto Press, 1972), 36 (nouvelle édition) et Terry Copp, Classe ouvrière et pauvreté (Montréal, Boréal Express, 1978), 31-32.

28 Registres de publications de bans, paroisse Sainte-Brigide, 22 mai 1912. 
de la lumière électrique et des bouquets. Malheureusement, c'est toute l'information qu'on a trouvée sur ce point.

En raison de la simplicité qui le caractérise, de l'heure et du jour auxquels la cérémonie se déroule, le mariage apparaît donc, au terme de cette courte étude, moins comme une consécration que comme une formalité administrative à laquelle la presque totalité des jeunes couples se soumettent, au printemps de préférence mais également à l'automne. Pour en acquitter les frais, le jeune homme débourse une grosse partie sinon la totalité de son salaire d'une semaine mais rarement davantage.

\section{Conclusion}

Sainte-Brigide est la seule paroisse montréalaise qui ait conservé, pour le début du 20e siècle, ses registres de publications de bans. Si cette source exceptionnelle ne permet pas de connaître la façon dont les noces étaient vécues en milieu ouvrier ni de saisir, dans son fonctionnement quotidien, les modes d'organisation familiale des travailleurs, elle contribue néanmoins à cerner d'un peu plus près les caractères de la cohésion sociale dans laquelle vivent les travailleurs brigidains et montréalais, à brosser le tableau de la structure du cadre de leur vie familiale et à préciser certains aspects de la cérémonie nuptiale. Peu de concubinages, peu de naissances illégitimes, peu de séparations volontaires des couples, bonne participation des jeunes filles à la congrégation des Enfants de Marie: autant d'indices qui permettent de conclure que les travailleurs brigidains et montréalais n'ont ni contesté, ni refusé le modèle familial proposé par l'Église, au contraire de l'attitude des ouvriers européens à l'époque de l'industrialisation ${ }^{29}$. Par contre leurs conditions de vie et de travail les ont amenés à développer des formes de solidarité entre parents et entre voisins susceptibles de les aider à compenser la faiblesse des salaires, l'incertitude de l'emploi et les limites de la charité publique et privée.

Même si l'histoire de la famille est un secteur de l'historiographie qui a connu depuis quelques années de grands développements dans plusieurs pays et a déjà effectué quelques percées importantes au Québec, l'étude de la famille ouvrière à l'époque de l'industrialisation de Montréal se heurte encore à de nombreuses difficultés, à cause principalement de la rareté et de la discrétion des sources. Il faut espérer que des travaux analogues soient conduits pour le milieu rural et pour les classes urbaines plus aisées afin de favoriser, par des comparaisons, une meilleure connaissance des structures générales de la famille québécoise à cette époque comme des spécificités qui marquent chaque classe.

\footnotetext{
${ }^{29}$ Voir, entres autres, Pierre Pierrard, op. cit., et particulièrement son chapitre 9, «L'attitude religieuse des ouvriers", 362-427.
} 
Mais au-delà de son intérêt pour elle-même, l'histoire de la famille constitue une voie privilégiée d'entrée pour l'étude de la vie quotidienne en milieu ouvrier et, plus généralement, pour celle de la culture ouvrière. Malgré quelques défrichages récents dans le domaine, les articulations de la culture ouvrière, ses dominantes, la vision du monde et la conception de l'existence qu'elle commande, restent encore à analyser et l'étude de la famille peut en être un des points de départ les plus fructueux. 